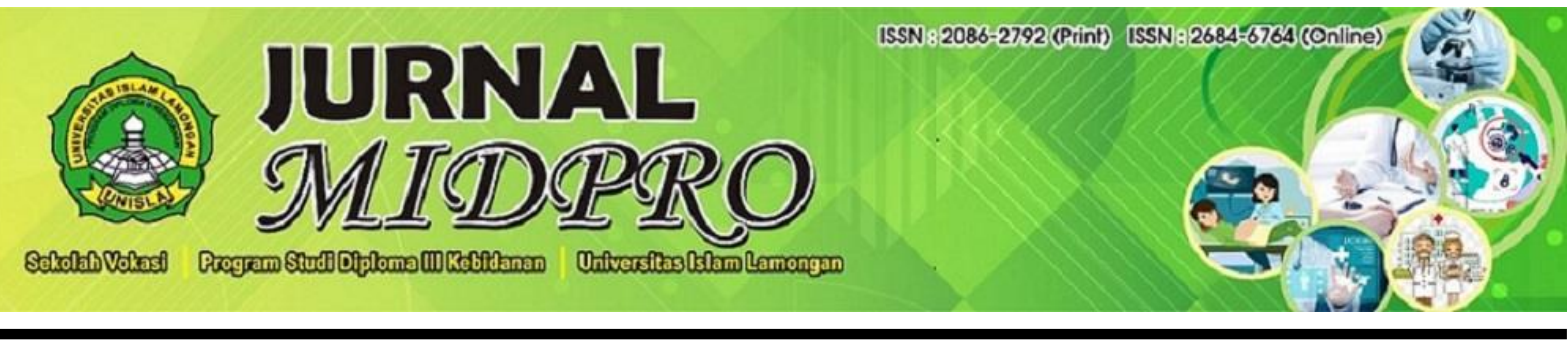

\title{
Antenatal Clinical Hypnosis And Birth Ball Exercise As Adjunct Therapy For Management Of Labor And Delivery Pain: A Summary Of Its Empirical Findings
}

\author{
${ }^{\text {KNi Gusti Ayu Pramita Aswitami }}{ }^{1}$, Ni Gusti Ayu Pitria Septiani ${ }^{2}$ \\ ${ }^{1}$ Department of Midwifery, Institute of Health Science Bina Usada Bali Badung, Bali Indonesia \\ ${ }^{2}$ Faculty of Medicine, Udayana University, \\ Email Korespondensi ( $\left.{ }^{\mathrm{K}}\right)$ : tami chant@yahoo.com
}

\begin{abstract}
Labor pain is conventionally defined similarly to acute pain and exhibits a complex constellation of multiple physiological and psychological factors. Pressure, anxiety, fear, feelings of self-efficacy, coping skills, and social support are influential factors regarding a woman's perception of pain and labor experiences. This pain varies greatly and often described as the most extreme pain that can be experienced or as agony or like torture that women may go through. Although epidural analgesia is generally accepted to be the gold standard method in intrapartum analgesia, there are risks associated with their use. Any less invasive but effective non-pharmacological approaches that can be used as an analgesic adjunct would be of great importance to the obstetric community. In recent years, clinical hypnosis and birth ball exercise have become an area of increasing clinical interest as methods to help the mother cope with the laboring process. They are frequently applicable, low-cost, and can be used as a supplementary treatment with other medications. Given widespread claims of efficacy, we aimed to review the available evidence regarding its effectiveness as an adjunct therapy for the management of labor and delivery pain. This is important as there is increasing interest among expectant parents and some health care providers about these methods for pain relief during the childbirth process.

While many of some investigations show positive effects on the outcome studied, in fact, there are conflicting findings in others showing no statistically significant of its benefits.
\end{abstract}

Keywords: adjunct therapy; birth ball; clinical hypnosis; labor pain; non-pharmacological methods

Article history :

Received: 17 Juni 2020

Received in revised form: 15 September 2020

Accepted: 10 October 2020

Available online: 1 December 2020

licensed by Creative Commons Attribution-ShareAlike 4.0 International License. 


\section{INTRODUCTION}

Pain management in labor was identified to be one the most important topics related to pregnancy and childbirth by about $78 \%$ of consumer groups and researchers. It represents a complex interaction of multiple physiological and psychological factors. Although labor pain is a physiological phenomenon during childbirth, it is usually painful and disquieting process for the most women. ${ }^{1}$ Nevertheless, management of labor pain is considered as a crucial aspect of obstetric care and considerable purpose of intra-partum care. Given the frequent adverse effects of pharmacological analgesia needs (i.e., epidural analgesia, opioids, and inhaled analgesia use nitrous oxide) for pain reduction during labor and the costly expenditures healthcare associated with their complications, there is an interest to use nonpharmacological pain relief methods for pregnant women before analgesic medications are accounted, particularly just before delivery. These methods can ease the pain sensations in respective ways, by boosting women's wellbeing, comfort, and sense of control in labor. ${ }^{1,2}$ In recent years, the clinical hypnosis and the birth ball exercise have become an area of increasing clinical interest as methods to help mother cope with the laboring process and childbirth. These non-pharmacological approaches are frequently applicable and low-cost, and can be used as a replacement or supplementary treatment with other medications. $^{2}$

Hypnosis is a field of study with a valuable clinical intervention as it is being increasingly used as part of psychological management of pain with regards to generous empirical evidence of its efficacy in the management of both chronic and acute pain. It is described as a natural state of being, existing 'when suggestion from one individual professedly alter the state of consciousness characterized by markedly increased of receptivity to suggestion, the extent of modification for perception and memories, as well as the capability for systematic control of a variety of common involuntary physiological functions.' There are some Cohhrane reviews have suggested the effectiveness of hypnosis for pain management especially in labor pain. ${ }^{1-3}$

In the review of the labor pain management studies, 25 RCTs were considered and 14 trials (including 749 women) satisfied the inclusion criteria. Women in the hypnosis group required less pharmacological intervention and might be increased the incidence of vaginal birth than in the control group. Additionally, there were no adverse effect to the mother or neonate recorded. The secondary benefits were associated with reducing the fear, tension, anxiety, and discomfort sensation before and during labor through inducing a focused state. ${ }^{1,3}$

More recently, Brown and Hammond in 2007 reviewed the benefits and efficacy of hypnosis in obstetric area. Hypnosis is proved to be an effective adjunct to the medical treatment of preterm labor in a case of quadruplets. Nevertheless, a high quality, multicenter randomized, clinical trial study is further recommended regarding the use of hypnosis in order to evaluate the efficacy of hypnosis in this area. Overall, the evidences study announce that hypnosis worthy as an adjunctive to other medical or psychological intervention by increasing and facilitating their efficacy and/or efficiency. Hypnosis may reduce the overall use of analgesia during labor, but not epidural use. ${ }^{5}$ 
The birth ball is also known as the fit ball, Swiss ball and Petzi ball, was firstly used in 1963 by physiotherapists for treating back pain. It was introduced as a childbirth tool in the 1980s by Perez and Simkin, who provided childbirth education to students, nurses, midwives and professional labor assistants. Perez stated that birth ball exercise was physically beneficial during pregnancy and labor. The systematic meta-analysis review have conducted in 2015 have critically evaluated the available evidence related to the impact of using a birth on labor pain relief. It has concluded that the clinical implementation of a birth ball exercise could be an effective tool for expectant women to alleviate labor pain. It is a low-cost intervention, which does not require complex training and could be valuable in low-resource region. Nonetheless, to reach a concrete conclusion, adequately powered RCTs or further high-quality researches to substantiate the efficacy of clinical hypnosis and birth ball are required with noticeable room for improvement within the research structure and methodology. ${ }^{2}$

\section{DEFINITION OF HYPNOSIS}

The hypnosis state is believed to be an altered state of consciousness characterized by remarkably increased of receptivity to suggestion, the capacity for modification of perception and memories, and with the capacity of systematic control for a variety of common involuntary physiological function. This technique typically involves a hypnotist and a person being hypnotized, but can also be selfadministered, i.e. as “self-hypnosis". It is accordance to Kihlstrom's definition of hypnosis in 1985 as "a social interaction offered by another person, designated the hypnotist, for experiences involving alteration in perception, memory, and voluntary action." Clinical hypnosis or hypnotherapy is an integrative mind-body skill of using words and gestures (hypnotic suggestions) during a focused mental state in order to achieve specific therapeutic clinical outcomes mutually described between a hypnotherapist and a client. ${ }^{1,6,7}$

The experience of the birth process is complex and demanding which influenced by various factors, including the labor duration, pain, medical interventions, anxiety, loss of control, support from partner and caregivers, and the woman's own expectations regarding the approaching childbirth. A negative and traumatic birth experience may increase the risk of post-traumatic stress syndrome, postpartum depression, bonding problems, and future cesarean section demand or refraining from having more children. ${ }^{7}$

The recent findings have reviewed implication of clinical hypnosis as a potential adjunctive to other interventions for helping expectant women manage and improve a wide range of psychological and pain sensation during labor. The most potential empirical evidence which Hitherto has found when clinical hypnosis is used as an complement to psychological and medical interventions. A review of Brown and Hammond in 2007 stated that hypnosis decreased fear, tension, and pain thus significantly reduced labor and delivery pain along with the need for analgesic medication during and after labor and delivery process. ${ }^{7-9}$ To the current review of one in a series of Cochrane Database of Systematic Reviews in 2016 
investigating the effectiveness and safety of hypnosis for pain management during labor and childbirth have independently extracted and assessed nine trials randomizing a total 2954 women. It has concluded that hypnosis may reduce the relative use of pharmacological analgesia during labor, but not epidural use (average RR $0.48,95 \%$ CI 0.32 to 0.73 , two studies, and 562 women). The hypnosis group were also more likely to have a spontaneous vaginal birth (RR 2.32, 95\% CI 1.43 to 4.07 ). However, there is not enough potential evidence to show whether hypnosis helps women feel more satisfied about their pain relief in labor, or whether it improves their sense of dealing with labor. Hence, further high-quality research is needed to prioritize the measurement of these outcomes. ${ }^{13}$

\section{PAIN DURING LABOR AND DELIVERY}

Labor pain is conventionally been defined similarly to acute pain, which is caused by uterine contractions, the dilatation of the cervix, and in the late first stage and second stage induced by stretching of the vagina and pelvic floor to accommodate the baby descends. The pain represents a complex constellation of multiple physiological and psychological factors (i.e., unpleasant sensory, perceptual and emotional experiences and certain autonomic, physiologic, emotional and behavioral responses). ${ }^{23}$ However, unlike any other acute pain, labor pain does not signal harm or pathologic process and considered as a normal part of birth. A major review by Lowe in 2002 noted that women's experiences of pain during labor are complex circumstance and even though almost expectant women receive some pain during childbirth, their sensory and affective perceptions can be very broadly. The sensations associated with labor pain have been described as among the most intense forms of pain that can be experienced to the extreme level which some women describe it as agony or like torture they may go through. However, the complete removal of pain does not necessarily mean a more gratifying birth experience for some women. Thus, clinical hypnosis is frequently considered by women interested in minimizing their chances of requiring a medical intervention during childbirth. ${ }^{11-14,24}$

\section{THE SENSORY AND AFFECTIVE COMPONENTS OF PAIN}

To the recent update, the neurophysiological studies have provided evidences supporting the theory that separate neuronal pathways are involved in distinction between the sensory and affective component of labor pain. The sensory dimension include the intensity, location and quality of the sensation of pain, while the affective components described as the emotional component, including elements of perceived unpleasantness of a stimulus. Price, Harkins, and Baker in 1987 have examined the specific character in relation to labor. Overall, they have 
found that women's ratings of affective pain were significantly lower than their ratings of sensory pain for all stages of labor pain except early labor (rated by both a visual analogue scale for pain sensation/VAS sensory and a visual analogue scale for degree of unpleasantness/ VAS affective). In addition, they also found that the women's affective, but not sensory ratings of pain were much lower when they reported they were more focused on the birth of their baby rather than on the pain sensations. Furthermore, in a thoughtful exploration of relations between pain and suffering has been proposed by Chapman and Gavrin in 1993 noted that potentially painful and horrifying situations can, under certain circumstances, be experienced as stimulating and exciting rather than as a cause of misery. Hence it was particularly relevant to the experience of pain in childbirth has been noted by Lowe in 2002 that psychological intervention such as having a support person in labor and childbirth preparation could be helpful for women, even if they did not reduce the sensory component of pain. ${ }^{14,24-26}$

\section{THE PSYCHOSOCIAL FACTORS ASSOCIATED WITH LABOR AND DELIVERY PAIN}

Bonica and McDonald in 1990 have noted that the uterine contractions dilating the cervix in the first stage of labor is a physiological processes, which are thought to cause pain during labor. Although pain intensity will obviously escalate with more frequent of uterine contractions and greater cervical dilation, these patterns are not consistently same across all women, but more related with the relationship between women's pain experiences and a range of physical factors including parity and maternal positions. Nulliparous women have consistently been found to experience greater pain intensity than multi-parous women in early labor state (cervical dilatation less than $5 \mathrm{~cm}$ ). Meanwhile, maternal positioning has also been found to effect women's experiences of labor pain and upright positions have been shown to have particular benefits in reducing of labor pain and epidural analgesia need in the first stage of labor. ${ }^{14,15,27}$

A wide range of psychosocial factors including anxiety, fear, feeling of self-efficacy, coping skills, expectations and social support have also been shown to impact upon women's experiences of labor pain. Lowe in 1996 have noted in spite of some anxiety is considered normal in labor, excessive anxiety leads to the release of additional stress hormones which can decrease blood flow to the pelvic area, increase muscular tension, thus amplify the pain stimuli and perception to the cortical brain. A Cochrane review has demonstrated that continuous support during labor had a positive impact to women's satisfaction feeling of their childbirth experience and use of pharmacological analgesia. They identified the key elements of this 
support comprise emotional support, information, suggestions of coping techniques, comfort and advocacy for the laboring woman. . $^{8,911,28}$

Researchers studying a group of Hungarian women in 1990 have found that primigravidas in the second and third trimester had a significantly higher hypnotizability scale by using the 12-point Harvard Group Scale of Hypnotic Susceptibility than non-pregnant women where 180 pregnant women scored an average 8.12 points, compared with the Hungarian average of 5.15 points. Primigravidas were more particularly hypnotizable, reaching an average of 9.0. ${ }^{13,29}$ Systematic review evidence of the effects of hypnosis for pain relief in childbirth suggest that hypnosis can decrease analgesia requirement during labor, decrease the use of oxytocin labor augmentation and increase the incidence of vaginal birth. In addition, a review study in 2004 has been conducted by Cyna et.al have suggested clinical hypnosis was associated with a reduction in the level of medical intervention in childbirth and associated risk to both mother and baby. As previous described, practically, hypnosis is best seen as an adjunct to facilitate patient care and enhance the effects of other analgesic. ${ }^{14,15,29,30}$

\section{BIRTH BALL EXERCISES AND POSTURE}

In recent years, advocacy to keep birth normal has shifted main point of the labor pain management towards midwifery model of care, which encompasses the psycho-emotional and spiritual component of care. There is a focus on building a woman's confidence to cope with pain rather than passive compliance, thus preserving a sense of mastery and well-being. The non-pharmacologic approaches with antenatal clinical hypnosis and birth ball exercise have become an area of increasing clinical interest and research as methods helping mothers to deal with laboring process and childbirth. ${ }^{31-33}$ The proper exercise during pregnancy strengthen the pelvic floor muscles by $90 \%$ which is essential for natural delivery. Birth ball is a new exercise tool which promotes pelvic rotation and increase the pelvic mobility. It is a large air filled rubber ball (approximately $60 \mathrm{~cm}$ in diameter) which is strong enough to support the mother's weight. ${ }^{33-35}$ The exercise is performed in the upright and sitting positions, which is considered to encourage delivery and promote the perineum to facilitate relaxation, and relieve labour pain. It also aid to widen and flex the pelvic bone and joints and accommodate baby to descent into birth canal more easily. Sekendiz et al. in 2012 stated that birth ball exercise may increase the strength, resistance, flexibility, and balance of the trunk, lower back, and quadriceps muscles. It also helps strengthen the pelvic diaphragm muscle, which responsible in pushing stage of the childbirth. ${ }^{34-36}$ Hau et al. in 2012 found that use of birth ball may alleviate pain and anxiety levels of women in labour process, furthermore the duration of the first stage of labor was 
significantly shorter in their study group. To sustain the training effects of the antenatal exercise classes and enhance the effectiveness of birth ball exercise, a collaboration team initiated by physiotherapist and midwife are deemed appropriately to teach the technique precisely and effectively. ${ }^{33,36-38}$ It has been applied in the study conducted by Lau et al. in 2013 as pioneer study of 203 primiparous or multiparous assigned as eligible sample study. Physiotherapist assessed the women's physical condition and taught birth ball exercises for 20-30 minutes, 3 times per weeks for 6-8 weeks after 34 or before 42 weeks of gestation in grouped session or even after the teaching session, whenever pregnant women felt like it. It is consisted of four main types of exercises, repeated in a 10 minutes interval, as follow: (1) sitting on birth ball (pelvic rocking-forward and back, Hula-hula- side to side, and rocking); (2) stand up (bend forward on the ball and leaning against the ball on the wall - up and down); (3) kneeling (hugging the ball and pelvic rocking); and (4) squatting (leaning against the ball on the wall). It suggested significantly reduced labor pain and back pain with the mean (SD) labor pain changed from 5.3 (2.6) before birth ball exercises to 4.3 (2.5), the mean reduction being 1.0 (95\% [CI], 0.8-1.3. $\cdot^{33,35,39-41}$ Most of studies measured labor pain relief by visual analogue scale (VAS) as a rapid measurement scale, the short form of the McGill pain (SF-MPQ) questionnaires, and in addition of the childbirth self-efficacy inventory (CBSEI). The secondary outcomes suggested it also promoted comfort and relaxation, and alleviating anxiety, the mean (SD) stress and anxiety levels change from 5.2 (2.9) before birth ball exercise to 3.7 (2.6) after; the mean reduction being $1.5(95 \% \mathrm{CI}, 1.3-1.8)$ which was statistically and clinically significant $(\mathrm{P}<0.001)^{35,36,42,43}$

A systematic review and meta-analysis of eight potential randomized controlled studies conducted by Makvandi et al. in 2015 has showed a statistically significant decrease in labor pain when a birth ball exercise was used in the first stage of labor. There are some potential mechanisms to explain this process. The first endogenous mechanism is gate control theory, which consists of applying non-painful messages to painful area. This mechanism has a main role in the sensory discriminative components of pain, by blocking part of the nociceptive message in the spine. According to this theory, the birth ball may grant supports of the perineum without applying significant pressure. ${ }^{32,44-46}$ Moreover, some studies indicated that freedom of movement and upright positions, including sitting in a rocking chair, on a birth ball or on the toilet during labor assisted the innate strength of gravity to facilitate and enhance fetal descent, improving the quality and effectiveness of labor contractions and decreasing labor pain. In addition, the decrease in labor pain that occurs with birth ball exercises might be attributed to distraction from pain. Distraction includes certain activities are equipped for expectant women 
so that conscious thoughts and anxieties can be reduced. During the contraction, the birth ball contribute to get rid of painful stimuli by distracting from the labor pain. ${ }^{32,34,47}$ However, in this systematic review has also noticed an issues from RCTs about the best timing of birth ball exercise should be commenced. In one trial, it was initiated in the prenatal period and continued during the first stage of labor. Therefore, it is necessary to isolate the effects of birth ball exercise during prenatal period from the first stage of labor in terms of labor pain reduction. Regarding the adverse effect in using birth ball during labor, the study of 40 primigravid mothers has been conducted by Rajakumari et al. in 2015 has demonstrated that use was safe. The main finding was a significant reduction of first stage of labor, thus it has been suggested that birth ball helped the baby find its best fit through the pelvis and facilitate fetal head rotation and descent, thus making labor shorter. ${ }^{41,48,49}$ Furthermore, recent quasi-experimental study was conducted by Fournier et al. in 2016 have found negative correlation between total training time on the birth ball exercise and total labor time ( $\mathrm{r}=-0.408 ; p=0.031)$, in other word, an increased training times with a birth ball during pregnancy was associated with shorter labor duration for both stage $1(\mathrm{r}=-0.372 ; p=0.043)$ and stage $2(\mathrm{r}=-0.415 ; p=0.028) .{ }^{50-52}$

The most of studies have their limitation in terms of including the concomitant use of other non-pharmacological pain relief during labor, but not specifically investigating the combination between clinical hypnoanalgesia and birth ball exercise itself. One of RCT has been conducted by Henrique et al. in 2016 assessed the influence of perineal exercise using birth ball in combination with warm bath or hypnotherapy. The effect of local heat during the bath stimulate redistribution of muscular blood flow, increasing relaxation and comfort. ${ }^{53-55}$ They evaluated this combination during the dilation phase, is associated with significant reduction of the labor pain and reduction of analgesia requirement, had a greater occurrence of vaginal delivery $(87.2 \%)$, and a more accelerating progress of cervical dilatation. Furthermore, it has suggested a better evolution of fetal presentation, increased frequency of contraction $(p<$ 0.001 ) and a greater reduction in labor times 41.8 minutes compared to the control. ${ }^{55-56}$

\section{LIMITATION OF THE PRESENT STUDY}

The findings evidence has showed a trend toward expectant women in the clinical hypnosis group being less likely to use the pharmacological pain relief or analgesia than those in the control group, although the result did not statistical significance. Confident intervals were very wide for all outcomes where data was available, indicating that further studies are needed to gain greater precision about the size of any effect. Additionally, adjustment of standard hypnosis technique, control of confounding variables, standardizing dependent measures, 
hypnosis susceptibility, blinding, allocation concealment, and power calculation of assessed outcomes are all issues that should be overcome in future studies. Furthermore, no trials have studied the economic involvement regarding hypnotherapy implication as part of routine clinical practice. Hence, suggestion should be made for incorporating cost-benefits analyses into the design for future studies.

In the other hand, some limitation of the birth ball studies included the concomitant use of the other non-pharmacological pain relief methods during labor. It is common for pregnant women to use several analgesic methods at the same time. Although the time and method of all the analgesic methods are noted, the outcome effects of using a birth ball would become difficult to measure. Furthermore, although the research protocol has been standardized, different hospital have different working cultures, staff attitudes, and setting, and hence this can lead to deviation in practice and therefore bias, hence meetings and training should be given to the physiotherapist and midwives at study sites before and periodically during recruitment.

\section{CONCLUSION}

Childbirth is a normal life event yet considered one of the most painful experience of a woman's life. Thus, management of labor pain is an indispensable aspect of obstetric care and a major goal of antenatal care. Epidural analgesia is a pharmacological approaches which has proven to be efficient in reducing pain during labor and now routinely used. However, its administration may frequently give adverse effects and disturb a mother's active cooperation during labor. Furthermore, it may expense high cost of healthcare associated with their complication. In recent years, the clinical hypnosis and birth ball exercise have become an area of increasing clinical interest to help mother cope with the laboring process and childbirth. These non-pharmacological methods of labor pain relief are frequently applicable and cheap, and can be used as a successor or supplementary treatment with other medications.

The current meta-analysis study by Madden in 2013 and Cochrane Database of Systematic Reviews in 2016 have included some high quality of RCTs in investigating the effectiveness and safety of hypnosis for pain management during labor and childbirth. It has showed a trend toward women in the hypnosis group being less likely to use the pharmacological pain relief or analgesia than those in the control group but not epidural use, although the result did not statistical significance and they were also more likely to have a spontaneous vaginal birth than control group. Meanwhile, no significant difference were found between women in the hypnosis group and the control group for the secondary outcomes of any effect on duration of childbirth, assisted vaginal birth, cesarean birth, or use of epidural/neuroxial block. There is currently not 
enough evidence to say whether hypnosis helps women feel more satisfied about their pain relief in labor, nor whether it improves their sense of coping with labor.

The effectiveness of birth ball exercise have demonstrated by some RCTs, which one of them $(n=188)$ demonstrated a $30-40 \%$ reduction in labor pain in interventional group. Women in the birth ball exercise group also had a shorter first stage (both of stage 1 and 2 for nulliparaous), less epidural requirement and fewer cesarean sections. There is, however, limited evidence in birth ball exercise use to prevent severe labour pain. The combination of birth ball exercise with warm bath showed more effective changes in the labor progress.

Overall, the findings of research indicate that clinical hypnosis and birth ball exercise used as an adjunctive to other medical intervention increases and facilitate the efficacy and/or efficiency of these interventions. However, to reach a concrete conclusion, adequately powered RCTs or further high-quality research to substantiate the efficacy of clinical hypnoanalgesia and birth ball are required with considerable room for improvement within the research structure and methodology.

\section{REFERENCES}

1. Cowen LW. How effective hypnosis for pain relief? Journal of Complementary Medicine. November, 2009; 8(6):18-20. Available at: https://www.researchgate.net/publication/297963296_Analgesic_hypnosis

2. Yeung, Tsang KWK, Yip BHK, et al. Birth ball for pregnant women in labour research protocol: a multi-centre randomized controlled trial. BMC Pregnancy and Childbirth. 2019; 19 (153): 1-6. DOI: 10.1186/s12884-019-2305-8.

3. Werner A, Uldbjerg N, Zacharie R, et al. Effect of self-hypnosis on duration of labor and maternal and neonatal outcomes: a randomized controlled trial. ACTA Obstetricia et Gynecologica Scandinavia. 2013; 92: 816-23. DOI: 10.1111/aogs.12141.

4. Mendoza ME, Capafons A. Efficacy of clinical hypnosis: A summary of its empirical evidence. Papeles del Psicólogo, 2009; 30(2): 98-116. Available at: https://www.academia.edu/14709599/EFFICACY_OF_CLINICAL_HYPNOSIS_A_SUMMARY _OF_ITS_EMPIRICAL_EVIDENCE

5. Brown DC, Hammond DC. Evidence-Based Clinical Hypnosis for Obstetrics, Labor and Delivery, and Preterm Labor. Intl. Journal of Clinical and experimental Hypnosis. 2007; 55(3): 355-371. DOI: $10.1080 / 00207140701338654$.

6. Lanfranco RC, Canales-Johnson A, Huepe D. Hypnoanalgesia and the study of pain experience: from Cajal to modern neuroscience. Frontiers in Psychology. September2014; 5 (1126): 1-7. DOI: 10.3389/fpsyg.2014.01126.

7. Patterson DR, Jensen MP. Hypnosis and Clinical Pain. Psychological Bulletin. 2003; 129 (4): 495521. DOI: 10.1037/0033-2909.129.4.495.

8. Cyna AM, Andrew MI, Robinson JS, et al. Study protocol: Hypnosis Antenatal Training for Childbirth (HATCh): a randomised controlled trial [NCT00282204]. BMC Pregnancy and Childbirth. 2006; 6(5): 1-12. DOI: 10.1186/1471-2393-6-5

9. Cyna AM, The Hypnosis Antenatal Training for Childbirth-University of Adelaide 1116160Revised Thesis. June, 2011. Available at: https://pdfs.semanticscholar.org/685c/3562903a8bdafc05800d08eea082b8dbfb01.pdf 
10. Kendrick C, Sliwinski J, Yu Y, et al. Hypnosis for acute procedural pain: A critical review. Intl. Journal of Clinical and experimental Hypnosis. 2016; 64(1): 75-115. DOI: 10.1080/00207144.2015.1099405

11. Vusse LV, Irland J, Berner MA, et al. Hypnosis for Childbirth: A Retrospective Comparative Analysis of Outcomes in One Obstetrician's Practice. American Journal of Clinical Hypnosis. October, 2007; 50(2): 109-19. DOI: 10.1080/00029157.2007.10401608.

12. Madden K, Middleton P, Cyna AM, et al. Hypnosis for pain management during labour and childbirth (Protocol). Cochrane Database Syst Rev. November, 2012; CD009356. DOI: 10.1002/14651858.CD009356.pub2

13. Madden K, Middleton P, Cyna AM, et al. Hypnosis for pain management during labour and childbirth (Review). Cochrane Database Syst Rev. 2016. DOI: 10.1002/14651858.CD009356.pub3

14. Madden K. Hypnosis for Pain Management during Childbirth: A Meta-analysis. Department of Psychology, University of Tasmania. 2012. Available at: https://pdfs.semanticscholar.org/fbfe/2711ffd8a49d954bb3bb5940945c797a7239.pdf

15. Cyna AM, McAuliffe GL, Andrew MI. Hypnosis for pain relief in labour and childbirth: a systematic review. British Journal of Anaesthesia. July, 2004; 94(4): 505-11. DOI: 10.1093/bja/aeh225.

16. Stewart JH. Hypnosis in Contemporary Medicine. Mayo Clin Proc. 2005; 80(4): 511-524. DOI: 10.4065/80.4.511.

17. Barber TX. The Effects of "Hypnosis" on Pain: A Critical Review of Experimental and Clinical Findings. Psychosomatic Medicine. 1963; 25: 303-33. Available at: https://pdfs.semanticscholar.org/aac0/cb4fb579c4640274cc8384ccc15c44ce985e.pdf

18. Beebe KR. Hypnotherapy for Labor and Birth. Nursing for Women's Health. February, 2014; 18(1): 48-59. DOI: 10.1111/1751-486X.12093

19. Burte JM. Hypnotherapeutic Advances in Pain Management. Psychology. 2002. Available at: http://drburte.com/docs/hypnosis-pain.pdf

20. Azizmohammadi S, Aszizmohammadi S. Hypnotherapy in delivery pain. Eur J Trans Myol. 2019; 29(3): 210-217.

21. Tomé Lopes Pires CdO. Pain and Hypnosis - Department of psychology, Universitat Rovira i Virgili - Dissertation Thesis. 2014. Available at: https://eg.uc.pt/bitstream/10316/79504/1/THESIS_DEF_Catarina\%281\%29.pdf

22. Abbasi M, Ghazi F, Barlow-Harrison A, et al. The Effect of Hypnosis on Pain relief during Labor and Childbirth in Iranian Pregnant Women. International Journal of Clinical and experimental Hypnosis. 2009; 57(2): 174-183. DOI: 10.1080/00207140802665435.

23. Thompson T, Terhune DB, Oram C, et al. The effectiveness of hypnosis for pain relief: A systematic review and meta-analysis of 85 controlled experimental trials. Neuroscience and Biobehavioral Reviews. 2019; 99: 298-310. DOI: 10.1016/j.neubiorev.2019.02.013.

24. Landolt AS, Milling LS. The efficacy of hypnosis as an intervention for labor and delivery pain: a comprehensive methodological review. Clin Psychol Rev. August, 2011; 31(6): 1022-31. DOI: 10.1016/j.cpr.2016.06.002.

25. Finalyson K, Downe S, Hinder S, et al. Unexpected consequences: women's experiences of a selfhypnosis intervention to help with pain during labour. BMC Pregnancy and Childbirth. 2015; 15(229): 1-9. DOI: 10.1186/s12884-015-0659-0.

26. Dugonjić M, Fajs N, Ivek I, et al. The Use of Hypnosis in Medicine: From Neuroscience to Clinical Practice. GYRUS. 2014; 4(2): 207-215. Available at: http://gyrus.hiim.hr/images/gyrus12/final web highq_Part8.pdf

27. De Pascalis V, Varriale V, Cacace I. Pain Modulation in Walking and Hypnosis in Women: EvenRelated Potentials and Sources of Cortical Activity. PloS One. 2015; 10(6): 1-38. DOI: 10.1371/journal.pone.0128474.

28. Chaillet N, Belaid L, Crochetié C, et al. Nonpharmacological approaches for pain management during labor compared with usual care: a meta-analysis. Birth. June, 2014; 41(2): 122-37. DOI: 10.1111/birt.12103.

29. Whitburn LY, Jone LE, Davey MA, et al. The nature of labour pain: An updated review of the literature. Women and Birth. April, 2018; 32(1): 28-38. DOI: 10.1016/j/wombi.2018.03.004. 
30. Reynold F. The effects of maternal labour analgesia on the fetus. Best Pract Res Cli Obstet Gynaecol. June, 2010; 24(3): 289-302. DOI: 10.1016/j.bpobgyn.2009.11.003.

31. Taavoni S, Charkamyani F, Hashemdabaghian F, et al. Effect of Pelvic Movement using Birth Ball and Listening to Nature sounds and Honey Syrup Consumption on Labor pain in Nulliparous Women: A Randomized Clinical Trial. World Family Medicine. 2018; 16(4): 97-102. DOI: 10.5742/MEWFM.2018.93348.

32. Makvandi S, Sadeghi R, Roudsari RL, et al. Effect of birth ball on labor pain relief: A systematic review and meta-analysis. Journal of Obstetrics and Gynaecology Research. November, 2015; 41(11): 1679-86. DOI: 10.1111/jog.12802.

33. Hau WL, Tsang SL, Man LSK, et al. The Use of Birth Ball as a Method of Pain Management in Labour. Hong Kong J Gynaecol Obstet Midwifery. 2012; 12:63-8. Available at: https://www.hkjgom.org/sites/default/files/pdf/v12-p63-birth-ball.pdf

34. Leung RWC, Li JFP, Leung MKM, et al. Efficacy of birth ball exercises on labour pain management. Hong Kong Med J. 2013; 19(5): 393-9. DOI: 10.12809/hkmj133921.

35. Mirzakhani K, Hejazinia Z, Golmakani N, et al. Effect of Performing Birth Ball Exercises during Pregnancy on Mode Delivery in Primiparaous Women. Journal of Midwifery and Reproductive Health. 2015; 3(1): 269-75. DOI: 10.22038/jmrh.2015.3562.

36. Kurniawati A, Dasuki D, Kartini F. Effectiveness of Birth Ball Exercise to Decrease Labor Pain in The Active Phase of The First Stage of Labor on The Primigravida Women. Indonesian Journal of Nursing and Midwifery. 2017; 5(1): 1-10. DOI: 10.21927/jnki.2017.5(1).1-10

37. Kwan WSC, Chan SW, Li Wh. The birth ball experience: outcome evaluation of the intrapartum use of birth ball. Hong Kong J Gynaecol Obstet Midwifery. 2011; 11(1): 59-64. Available at: https://pdfs.semanticscholar.org/d4fa/0a4abc874345c61984de5ad74db846f88b9b.pdf

38. Delgado-García BE, Orts-Cortés MI, Poveda-Bernabeu A, Cabellero-Pérez P. Randomised controlled clinical trial to determine the effects of the use of birth ball during labour. Enferm Clin. 2012; 22(1): 35-40. DOI: 10.1016/j.enfcli.2011.07.001.

39. Sawyer A, Ayers S, Abbott J, Gyte G, et al. Measures of satisfaction with care during labour and birth: a comparative review. BMC Pregnancy Childbirth. 2013; 13:108. DOI: 10.1186/1471-239313-108.

40. Hodnett ED. Pain and women's satisfaction with the experience of childbirth: a systematic review. Am J Obstet Gynaecol. May, 2002; 186(5 Suuppl Nature): S160-72. DOI: 10.1067/mob.2002.121141

41. Rajakumari AG, Soli TK, Malathy D. Evaluation of the effectiveness of selected intervention in reducing level of pain perception and birth experience among primi gravida mothers. International Journal of Advances in Scientific Research. 2015; 1(04): 182-85. DOI: 10.7439/ijasr.

42. Taavoni S, Abdolahian S, Haghani H, et al. Effect of birth ball usage on pain in the active phase of labor: a randomized controlled trial. J Midwifery Womens health. 2011; 56(2): 137-40. DOI: 10.1111/j.1542-2011.2010.00013.x.

43. Brown ST, Douglas C, Flood LP. Women's evaluation of intrapartum nonpharmacological pain relief methods used during labor. J Perinat Educ. 2001; 10(3): 1-8. DOI: 10.1624/105812401X88273

44. Silva LM, Oliveira SMJV, Silva FMB, et al. Using Swiss ball in labor. Acta Paul Enferm. 2011; 24(5): 656-62. DOI: 10.1590/S0103-21002011000500010

45. Gau M-L, Chang C-Y, Tian S-H, et al. Effects of birth ball exercise on pain and self-efficacy during childbirth: A randomised controlled trial in Taiwan. Midwifery. 2011; 27: e293-e300. DOI: 10.1016/j.midw.2011.02.004.

46. Adams ED, Bianchi AL. A practical approach to labor support. J Obstet Gynecol Neonatal Nurs. 2008; 37(1): 106-115. DOI: 10.1111/j.1552-6909.2007.00213.x.

47. Mathew A, Nayak S, Vandana K. A Comparative study on Effect of Ambulation and Birthing Ball on Maternal and Newborn outcome Among Primigravida Mothers in Selected Hospital in Mangalore. Nitte University Journal of Health Science. 2012; 2(2): 2-5. Available at: http://nitte.edu.in/journal/juneSplit/Nitte\%20University\%20Journal\%20June\%202012_2_5.pdf

48. Lewis B, Avery M, Jennings E. The Effect of Exercise During Pregnancy on Maternal Outcomes: Practical Implication for Practice. American Journal of Lifestyle Medicine. 2008; 2(5): 441-55. DOI: $10.1177 / 1559827608320134$. 
49. Shallow H. My rolling programme the birth ball: ten year experience of using the physiotherapy ball for labouring women. MIDIRS Midwifery Digest. 2003; 13(1): 28-30.

50. Hodnett ED. Pain and women's satisfaction with the experience of childbirth: a systematic review. Am J Obstet Gynaecol. May, 2002; 186(5 Suppl Nature): S160-72. DOI: 10.1067/mob.2002.121141

51. Shirazi MG, Kohan S, Firoozehchian F, et al. Experience of Childbirth with Birth Ball: A Randomized Controlled Trial. International Journal of Women's Health and reproduction Sciences. July, 2019; 7(3): 301-305. DOI: 10.15296/ijwhr. 2019.50.

52. Fournier D, Feeney G, Marie-Eve M. Outcomes of exercise training following the use of a birthing ball during pregnancy and delivery. Journal of Strength and Conditioning Research. July, 2017; 31(7): 1941-47. DOI: 10.1519/JSC.0000000000001672

53. Davies GA, Wolfe LA, Mottola MF, et al. Joint SOGC/CSEP clinical practice guideline: exercise in pregnancy and the postpartum period. Can J Appl Physiol. June, 2003; 28(3): 330-41. DOI: $10.1139 / \mathrm{h} 03-024$

54. Zwelling E, Johnson K, Allen J. How to implement complementary therapies for labouring women. MCN Am J Matern Child Nurs. 2006; 31(6): 364-70. Available at: http://www.embase.com/search/results?subaction=viewrecord\&from=export\&id=L44878761

55. Henrique AJ, Gabrielloni MC, Cavalcanti ACV, et al. Hydrotherapy and the Swiss ball in labor: randomized clinical trial. Acta Paul Enferm. 2016; 29(6): 686-92. DOI: 10.1590/19820194201600096

56. Alleemudder DI, Yemi K, Kuponiyi C, et al. Analgesia for labour: an evidence-based insight for the obstetrician. Royal College of Obstetricians and Gynaecologists. 2015; 17: 147-55. DOI: 10.1111/tog. 12196 